plain, the extensive and varied alluvial territory that lies between Lakes Kivu and Tanganyika. The physical environment, the flora, the several habitats - some nine, ranging from aquatic to forest paraclimax, are indicated-and their floristic associations are described in detail and illustrated by graphs and numerous excellent photographs. The plain is floristically rich, with 877 species of seed plants and pteridophytes, comprising 427 genera and 99 families. There is a marked dominance of species of the basic Sudanese-Zambesian flora $(42 \cdot 6$ per cent) with 6 per cent of foreign species, chiefly from the west, the balance being transitional and widely distributed species.

\section{Electron Microscope and Microdissection}

UNder this title, R. Bovey (J. Roy. Micro. Soc., Ser. III, 72, 56 ; 1952) has described and illustrated a new technique for preparing biological specimens for study with the electron microscope. Using the de Fonbrune micromanipulator and an inverted microscope, the microdissection is made directly on a collodion film, or a film of 'Formvar', supported by a thin coverslip. The specimen is afterwards mounted on a grid. It is shown that the technique described can be successfully applied to the study of nuclear and cellular membranes and similar materials.

\section{Microbial Growth and its Inhibition}

Eighteren of the papers presented at the First International Symposium on Chemical Microbiology, which was held in Rome during June 1951, have been brought together under the title "Microbial Growth and its Inhibition" in No. 10 of the World Health Organization Monograph Series (pp. 285. Geneva : W.H.O. ; London: H.M.S.O., 1952. 15s., 3 dollars or $12 \mathrm{Sw}$. francs); the papers had been previously published in the Bulletin of the World Health Organization. Many of the communications deal with the production, chemistry and testing of antibiotics, while problems of resistance are discussed by $M$. Welsch and L. L. Cavalli. The lag phase in the growth of Pasteurella pestis is investigated by S. S. Sokhey, and B. C. J. G. Knight discusses the physiological background to problems of growth inhibition. The role of the B group of vitamins in biosynthetic processes is dealt with by $\mathrm{D}$. D. Woods, with particular reference to the metabolic functions of folic acid and $\mathrm{B}_{12}$, and $\mathrm{J}$. Monod presents his investigations concerning the specific inhibitors and inducers involved in the production of $\beta$-galactosidase in Escherichia coli. The collection of papers represents in general a number of contributions on specific aspects of microbial growth and of the properties of substances inhibiting such growth, rather than an outline or synthesis of present knowledge. Discussions are not published, but each paper concludes with useful summaries in both English and French.

\section{Centrifuges and $\mathrm{pH}$ Meters}

A suRvex of the requirements of biologists using centrifuges and $p \mathrm{H}$ meters has been made by the Institute of Biology. The analyses of the views expressed are given in two reports, which have been sent to all who collaborated in the survey and to all British manufacturers of the instruments. It is hoped that this presentation of the collected views of biologists, by making clear those features which are desirable but often lacking and the defects now experienced by users, will enable manufacturers to introduce the necessary modifications to existing models and to design future instruments to meet the expressed needs. Copies of the reports can be obtained from the General Secretary, Institute of Biology, Tavistock House South, Tavistock Square, W.C.l.

\section{A.S.T.M. Index of X-Ray Powder Diffraction Data}

THE index, prepared by the American Society for Testing Materials, of X-ray powder diffraction data, widely known as the A.S.T.M. Index, has now been in use for about ten years, and it is recognized that it contains a number of wrong identifications which are likely to lead to confusion. The X-ray Analysis Group of the Institute of Physics therefore proposes to collect information on the errors which have been observed by users of the Index, and to publish as soon as possible in some suitable way a list of all those errors which seem to be substantiated. It is believed that many, if not all, of these errors have by now been noted by various workers and may in some cases have been mentioned casually in their publications ; but in order to be of real use to others it is necessary that they should all be published collectively. Accordingly, everyone who has noted such errors is asked, whether he has already published them or not, to send the details to Dr. A. J. C. Wilson, University College, Cathays Park, Cardiff.

\section{Development of Mineral Resources in the United States}

A PANEL of specialists in geology, mining and geophysics representing industry, government and the universities in the United States has been established to advise the National Science Foundation on policy questions connected with the recommendations of the President's Materials Policy Commission for development of the mineral resources of the nation. The first meeting of the panel was held in Washington, D.C., on November 29, 1952 . In its report, the Commission recommended that "an intensive program of basic scientific research and technical development be undertaken on techniques and instruments of exploration for minerals", and the advisory panel of the Foundation will consider the need for an inventory of current research in exploration techniques and the formation of a committee to make such an inventory.

\section{Nineteenth International Physiological Congress, Montreal}

The Nineteenth International Physiological Congress will be held in Montreal during August 31September 4, 1953, and one of the main concerns of the organizers of the Congress is to ensure that an adequate number of scientific workers from overseas countries will attend, despite the relatively heavy financial expenses that must necessarily be incurred by such participants. Physiologists, biochemists, pharmacologists and others from outside Canada and the United States who hope to attend the Congress are therefore asked to notify the secretaries of their national societies of that fact as soon as possible. This will aid the Congress organizers in arranging for special lectures and discussions to be held in the United States and Canada during the weeks preceding and following the Congress; it is hoped that those from overseas who are invited to take part in such functions will, in some cases at least, receive financial help that will appreciably reduce the cost of attending the Congress itself. Those who live in countries where there are no national scientific societies, and who hope to attend the Congress, should write directly to the executive secretary of 